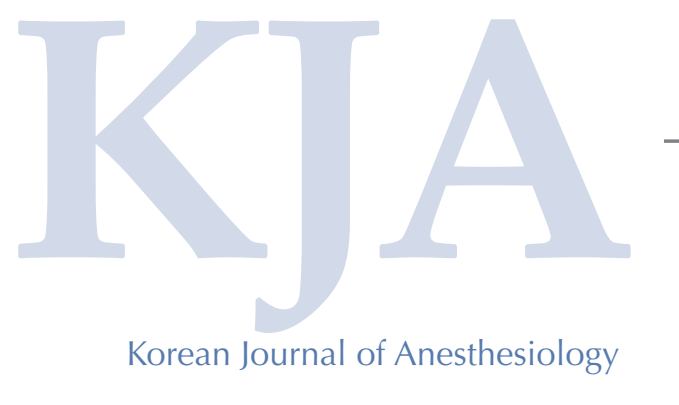

Editorial

\title{
The first step to good clinical research: statistical thinking
}

\author{
Sangseok Lee \\ Department of Anesthesiology and Pain Medicine, Sanggye Paik Hospital, Inje University College of Medicine, \\ Seoul, Korea
}

The Korean Journal of Anesthesiology, the official journal of the Korean Society of Anesthesiologists, recently introduced the Statistical Round as a new publishing category for the year 2015. The Aims and Scope section of the journal explains that the Statistical Round category provides educational material to help the reader understand the designs of experimental and statistical analyses used in anesthesiology investigations. A Statistical Round is a narrative review of the application of various topics ranging from contemporary quantitative sciences to issues of concern to anesthesia researchers. A Statistical Round provides a focused discussion of one or more unique or interesting statistical analysis methods that were previously published in this journal or express the general policies or opinions of the Statistical Round Board. Statistical Round articles are provided by members of the Statistical Round Board or are invited from authors and then are reviewed by the Statistical Editor. All of the Statistical Round articles are published in both English and Korean for the convenience of Korean readers; the Korean version is present only on the journal's web page.

Statistics is the practice or science of collecting and analyzing numerical data in large quantities, especially for the purpose of inferring proportions in a whole population from those in a representative sample. Statistical Round articles ultimately provide guidance for performing statistical analysis, which could be very helpful for both authors and reviewers. A good example of these goals can be found in other medical science journals (e.g., British Medical Journal, Anesthesia \& Analgesia) [1].

Corresponding author: Sangseok Lee, M.D.

Department of Anesthesiology and Pain Medicine, Sanggye Paik

Hospital, Inje University College of Medicine, 1342, Dongil-ro,

Nowon-gu, Seoul 130-707, Korea

Tel: 82-2-950-1171, Fax: 82-2-950-1323

E-mail: s2248@paik.ac.kr

ORCID: http://orcid.org/0000-0001-7023-3668

Korean J Anesthesiol 2015 April 68(2): 99-100

http://dx.doi.org/10.4097/kjae.2015.68.2.99
Many data obtained through research are questioned. How great should the difference in means between two samples be to consider the presence of a meaningful difference between the two populations? If the sample mean does not represent the average of the population, it is correct to analyze data using the t-test in such cases? What nonparametric methods correspond to one-factor analysis of variance (ANOVA)? When the null hypothesis is rejected after one-factor ANOVA, how can we determine whether all means are different between each groups? What would the result be of ignoring the nature of the repeatedmeasure data if an independent t-test is used to compare two groups? Can we only use continuous or categorical data to measure the level of the dependent variable in multiple regression analysis? Can we measure the levels of independent variables using both continuous and categorical data? Are any problems evident between the relevant independent variables included in the model? These are examples of questions requiring further investigation.

Indeed, even leading medical journals have not provided answers to these questions, sometimes resulting in incorrect statistical analyses [2,3]. Statistical Round articles will provide exemplary applications of practical solutions to these questions.

Randomization, operation, and control are generally investigated when evaluating the quality of research in evidence-based medicine. Therefore, randomized controlled trials are important because they can provide the highest level of evidence. The reliability of clinical studies for reference during care of patients in clinical practice is important. Clinicians should be able to rely on the results of clinical studies in their decision-making process. Although research ethics are expected to be followed and the research process is expected to be carried out honestly, clinical doctors, public health policy-makers, and others can only determine the reliability of a study through published papers. Therefore, the medical literature should clearly describe the results of clinical research that have been performed transparently and reliably. An excellent-quality paper should include welldescribed methods and conclusions. Even when vast numbers

(c) This is an open-access article distributed under the terms of the Creative Commons Attribution Non-Commercial License (http://creativecommons.org/ licenses/by-nc/3.0/), which permits unrestricted non-commercial use, distribution, and reproduction in any medium, provided the original work is properly cited. 
of clinical papers are being published, the value of the research cannot be properly assessed if the methodology and analysis are not adequately described, no matter how good the content of the study itself $[4,5]$.

As the first manuscript in the Statistical Round category, the work of Drs. Kang and Lee in this issue of the Korean Journal of Anesthesiology serves as a good guideline for the majority of researchers with these concerns and challenges [6]. The authors have described in detail how to set goals and hypotheses for a study, explain various points to consider in the study design, and describe the correct methods by which to explain the research process. Unfortunately, the authors were unable to provide numerous examples of actual cases for enhanced reader understanding. However, the checklist provided in the article gives readers an opportunity to evaluate studies themselves.

The Statistical Round section includes essays on statistics, but its content does not resemble that of a statistical textbook. Most people unfamiliar with statistics tend to believe that the field of statistics involves simple numerical data and statistical techniques for processing these data. However, this is only a very small part of statistics. Statistics is a process that cannot lead to the conclusion that to avert a logical deletion. It is not an exaggeration to say that statistical thinking may support the thought of modern people. A misunderstanding is that learning statistics simply involves learning how to analyze data and utilize various analytical methods. However, statistics are logically presented the pertinent thinking, and should be understood as a tool that makes this type of thinking possible. Statistical analyses need to logically explain the results of the study and provide a reasonable conclusion; therefore, the research methodology and interpretation of the results should be based on statistical thinking. Statistical literacy has become a necessity for the majority of contemporary researchers. The amount of data that can be obtained from patients has increased substantially with the development and dissemination of monitoring equipment. Timely analysis of clinical data and performance of effective treatment are important, and the ability to accomplish these goals is dependent upon one's ability to think statistically when evaluating these clinical data. The same is true when assessing researchobtained data.

It is not an exaggeration to say that statistical thinking holds the answer to determining whether a given paper is obsolete or a valuable tool. Thus, statistical thinking might be the first and last step in performing high-quality clinical research.

\section{References}

1. Dexter F. Checklist for Statistical Topics in Anesthesia \& Analgesia Reviews. Anesth Analg 2011; 113: 216-9.

2. Avram MJ, Shanks CA, Dykes MH, Ronai AK, Stiers WM. Statistical methods in anesthesia articles: an evaluation of two American journals during two six-month periods. Anesth Analg 1985; 64: 607-11.

3. Altman DG. Statistical reviewing for medical journals. Stat Med 1998; 17: 2661-74.

4. Shin C, Han C, Pae CU, Patkar AA. Tools for quality evaluation of clinical research reports. Korean J Psychopharmacol 2011; $22: 67-72$.

5. Plint AC, Moher D, Morrison A, Schulz K, Altman DG, Hill C, et al. Does the CONSORT checklist improve the quality of reports of randomised controlled trials? A systematic review. Med J Aust 2006; 185: 263-7.

6. Lee S, Kang H. Statistical and methodological considerations for reporting RCTs in medical literature. Korean J Anesthesiol 2015; 68: 10615. 